\title{
Experiencias de docencia, extensión e investigación para promover la lactancia y la donación de leche materna
}

\author{
Carla Estefanía Martin, Carina Lorena Fernández y Mara Cristina Romero
}

\begin{abstract}
Resumen
Actualmente las actividades de extensión ofrecen a los miembros de la comunidad universitaria vías de interacción con la sociedad, brindando herramientas y conocimientos que tienden a mejorar la realidad social en que sus actores despliegan sus prácticas. De esta manera, el objetivo de este artículo es presentar los resultados parciales de un trabajo que integra prácticas de extensión, docencia e investigación, en el marco de un Proyecto de Extensión Socio Comunitario (PESc), denominado "Lactancia materna y donación de leche Humana en la Unidad Médica Educativa de la Universidad Nacional del Chaco Austral". Dicho trabajo se llevó a cabo durante el año 2018. Nuestra experiencia fue positiva y aún continua en ejecución, ya que generó nuevos proyectos de extensión que se están llevando a cabo actualmente, además de la creación de una nueva línea de investigación. Con este texto esperamos transmitir nuestra experiencia y logros obtenidos.
\end{abstract}

Palabras clave: actividades de extensión, lactancia materna, donación de leche humana, banco de leche humana, promoción de la salud.

\section{TEACHING, EXTENSION AND RESEARCH ACTIVITIES FOR PROMOTING BREASTFEEDING AND BREAST MILK DONATION}

\begin{abstract}
Currently outreach extension activities offer members of the university ways of interacting with society, providing tools and knowledge that tend to improve the social reality in which its actors deploy their practices. Therefore, the objective of this article is to present the partial results of a work that integrates extension, teaching and research practices, within the framework of a Socio-Community Extension Project (PESC), named "Breastfeeding and Human Milk Donation in the Educational Medical Unit of the National University of Chaco Austral". This work was carried out during 2018. Our experience was positive, and it is still ongoing, since it generated new extension projects that are being carried out, in addition to the creation of a new line of research. With this paper we hope to convey our experience and achievements.
\end{abstract}

Keywords: extension activities, breastfeeding, donation of human milk, human milk bank, health promotion. 


\section{Carla Estefanía Martin}

martincarlaestefania@gmail.com orcid.org/0000-0002-1984-513X

Jefe de Trabajos Prácticos. Asignatura: Biología, de la carrera de Licenciatura en Nutrición. Jefa del Servicio de Nutrición de Pediatría de la Unidad Médica Educativa de la Universidad Nacional del Chaco Austral.

\section{Carina Lorena Fernández}

fernandezcarina@uncaus.edu.ar orcid.org/0000-0003-2326-4589

Profesora Adjunta. Cátedra: Fisicoquímica de la Carrera Ingeniería Química, de la Universidad Nacional del Chaco Austral. Categoría III en el Programa Nacional de Incentivos. Directora de proyectos de investigación y tesis de posgrado.

\section{Mara Cristina Romero}

mara@uncaus.edu.ar orcid.org/0000-0001-9624-9051

Profesora Adjunta. Cátedra: Industrias Alimentarias ॥, de la Carrera Ingeniería en Alimentos, de la Universidad Nacional del Chaco Austral. Investigador Asistente conicet. Categoría II en el Programa Nacional de Incentivos. Directora de proyectos de investigación y tesis de posgrado. 


\section{Introducción}

La información brindada desde la universidad contribuye al conocimiento de distintos temas y al bienestar de la comunidad, cumpliendo con uno de los compromisos sociales más importantes que es la promoción de la salud a través de prácticas voluntarias y solidarias. Es así que, desde hace ya algunos años, la extensión universitaria, también conocida como vinculación comunitaria, ha adquirido un rol muy importante en el ámbito universitario, permitiendo a la comunidad en su totalidad (docentes, no docentes, alumnos y graduados de distintas carreras) relacionarse entre sí (Ambrústolo, Migueles, Berardi y Zárate, 2018) e interactuar con quienes, en principio, volcarán sus conocimientos. Las problemáticas con las actividades de extensión deben buscarse junto con la comunidad destinataria y en su propio terreno, ya que, de otra manera, los sesgos de información pueden llevar a errores de identificación y malinterpretación de sus necesidades (Marín, 2016).

La extensión universitaria es una de las tres funciones sustantivas de la universidad, asociándose este concepto a cualquier actividad que, sin ser propiamente investigación o enseñanza curricular, se realice en vinculación con actores sociales no universitarios, como organismos estatales, empresas, organizaciones sociales y comunidades (Gezuraga, M., 2017; Cano Menoni y Castro Vilaboa, 2016). Esto lleva a que frecuentemente se considere que la extensión e investigación son actividades que están siempre separadas, cuando en realidad la integración de ambas, junto con la docencia, podría generar un enriquecimiento de todo el proceso de extensión. Esto se produciría ya que cada participante, desde su rol, aporta con sus conocimientos, y la asociación de éstos favorece el proceso, durante la realización y al momento de la retroalimentación. Por lo tanto, la extensión es uno de los factores dinamizadores de la misión universitaria y representa un modelo de proyección social que significa un beneficio para la comunidad y, además, una poderosa herramienta pedagógica para desarrollar actitudes solidarias y potenciar la virtud cívica entre los estudiantes (Marín, 2016).

Las actividades de extensión universitaria son muy frecuentes en carreras relacionadas al campo de la salud (López, 2016). Por ejemplo, en la Universidad Nacional del Chaco Austral (uncaus), en las carreras de Medicina y Nutrición, se aprovechan las prácticas de vinculación comunitaria para que sus alumnos puedan desarrollar experiencias que les permitan enriquecer las habilidades y conocimientos teóricos que poseen, al evaluar el estado de situación de procesos sociales y plantear soluciones posibles ante ciertos problemas de la comunidad. En este contexto surge el presente artículo, en el que se presentan los procesos y resultados parciales de un trabajo que integra prácticas de extensión, docencia e investigación, en el marco de un Proyecto de Extensión Socio Comunitario (PESC), denominado "Lactancia materna y donación de leche", llevado a cabo durante el año 2018. 
Si bien, en su inicio, el proyecto de partida buscó verificar el bajo nivel de conocimientos que poseen las madres respecto a la lactancia -para así concientizar a la población sobre la importancia de ésta y de la donación de leche para la alimentación y mantenimiento de la salud de infantes-, se indagó luego sobre las creencias respecto a la donación de leche a los Bancos de Leche Humana (BLH), con miras a la implementación de un Banco de Leche Materna (BLm) en la Unidad Médica Educativa de la Universidad Nacional del Chaco Austral. Docentes, estudiantes y graduados de las carreras de Medicina, Licenciatura en Nutrición e Ingeniería en Alimentos, que se dictan en esta casa de estudios, conforman el equipo de trabajo de los proyectos antes mencionados. En este artículo se presentarán algunos conceptos que sirvieron de base para el desarrollo del trabajo del PEsc, la metodología elegida para las actividades de campo, los resultados obtenidos, y las experiencias de los estudiantes participantes.

\section{Lactancia materna exclusiva y bancos de leche materna}

La Lactancia Materna Exclusiva (LME) es la forma más segura de brindar un alimento ideal para el crecimiento y desarrollo sano de un ser humano. Se recomienda a nivel mundial que se realice de manera exclusiva hasta los seis meses de vida (OMs/UNICEF, 2008). Esto significa que el lactante sólo debe recibir leche del pecho de su madre o de una nodriza, o bien a través del biberón con leche materna extraída del pecho, en los primeros meses de vida, y que esto se puede extender hasta los dos años de edad, complementando la leche con otro tipo de alimentos. No obstante, existen estudios que evidencian que, a pesar de que la mayoría de las madres conoce la necesidad de la lactancia materna hasta los dos años (Injante Injante, Álvarez Díaz, Gavilano Bernaola y Barriga, 2017; Aquino Rojas, 2018), la prevalencia de la misma es baja, por lo que se requiere de intervenciones tendientes a asegurar esta práctica. En nuestro país, en su informe del año 2015 el Ministerio de Salud de la Nación señaló una preocupante cantidad de niños destetados tempranamente, siendo muy pequeña la proporción de éstos que recibe Lme hasta el sexto mes. El destete precoz suele ir asociado a factores socio-culturales, como prejuicios personales, falta de apoyo familiar, condiciones laborales que no brindan espacios para esta práctica, actitudes con respecto a amamantar en lugares públicos y también falta de apoyo por parte de los profesionales de la salud quienes muchas veces no se preocupan por enseñar técnicas sobre cómo hacerlo (Ministerio de Salud de la Nación, 2015; Hernández Pérez, Díaz-Gómez, Romero Manzano, Díaz Gómez, Rodríguez Pérez y Jiménez Sosa, 2018). Por otra parte, existen casos en que la lactancia materna no es posible (como por ejemplo por enfermedad de la madre o el bebé), por lo que se recomienda la leche proveniente de una nodriza sana para la adecuada alimentación del niño, sin la necesidad de recurrir a un sucedáneo de leche materna. En caso de no contar con una nodriza, o en casos de bebés prematuros, que en general nacen con muy bajo peso, o en el caso de niños con patologías del aparato digestivo, o que atraviesan un postquirúrgico ( sin acceso a leche de su mamá), por lo que no pueden ser amamantados, la 
Figura 1. Distancia entre las ciudades de Presidencia Roque Sáenz Peña y Resistencia. Fuente: http:// www.argentinadistancia.com/ distance/30008656-30001968 alternativa de alimentación más adecuada es la proporcionada por un BLm, que es un centro especializado responsable, de la recolección, análisis, procesamiento, almacenamiento y distribución de leche humana donada a los pacientes que la necesiten, bajo prescripción médica, garantizando la calidad nutricional y la seguridad microbiológica y toxicológica de su consumo, encargándose además de actividades de promoción y apoyo a la lactancia materna, visualizada en nuestro país a través de la Ley Nacional N²6.873, de Promoción y Concientización Pública sobre la Lactancia Materna (Flores Antón, Alonso Díaz y García Lara, 2015; Delgado Panta, 2011; Herrera, Berganza, Giménez, Cardozo, y Jiménez, 2013). Desde 2011 nuestro país cuenta con la Comisión Técnica Asesora en Bancos de Leche Materna, a partir de la cual se crearon BLm en diferentes puntos estratégicos (Sager, 2018). Nuestra provincia, Chaco, cuenta con un BLm situado en el Hospital Dr. Julio C. Perrando de la ciudad de Resistencia, y una extensión del mismo ubicada en el Hospital 4 de Junio de nuestra localidad (Presidencia Roque Sáenz Peña), cumpliendo funciones únicamente de lactario, es decir, las madres acuden allí para la extracción de leche que posteriormente es trasladada a Resistencia para su tratamiento y análisis; y luego, esta leche tratada regresa al lactario para poder ser suministrada a los recién nacidos. Si bien este sistema funciona, la principal desventaja que tiene es la distancia que separa a ambos centros de salud (160 Kilómetros) y que, por ende, recorre la red láctea, como se muestra en la figura 1.

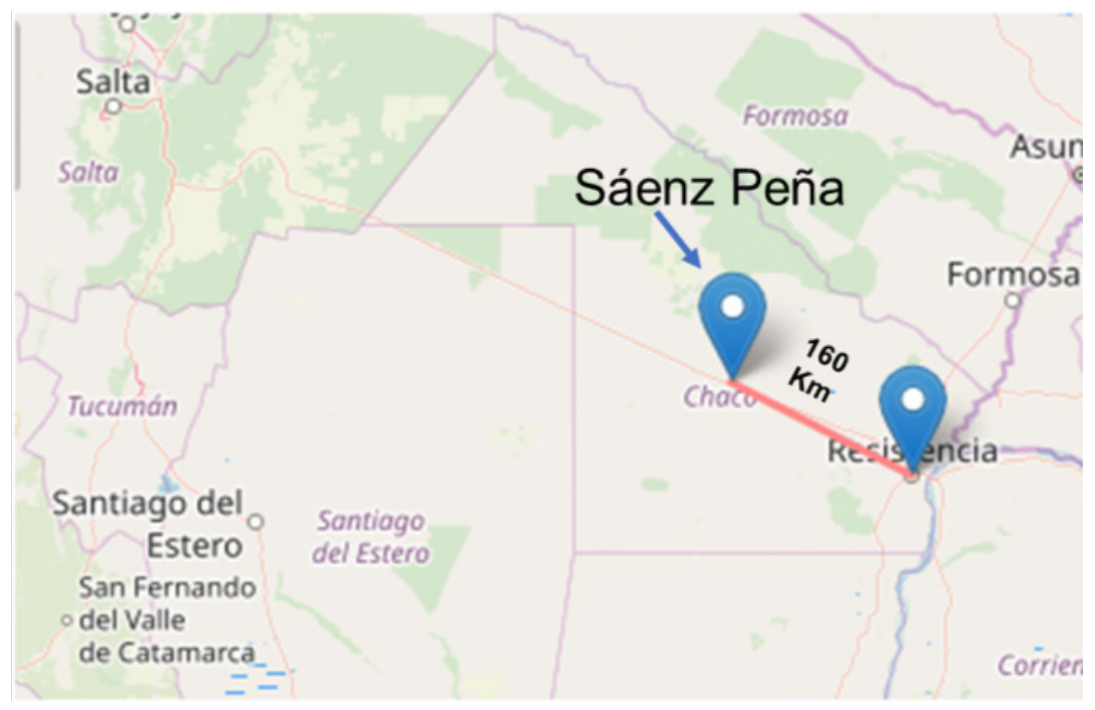

El inconveniente de la distancia entre el lactario de nuestra localidad y el BLm de la provincia ponen de manifiesto la necesidad y conveniencia de crear en nuestra región un BLM. En este sentido, nuestra universidad cuenta con la Unidad Médica Educativa (UME), la cual a partir de 2019 ha puesto en funcionamiento un lactario con miras a la implementación de un BLm propio, que servirá como unidad de apoyo clínico al Servicio de Neonatología que funciona en dicha unidad. La Ume es un centro de salud ubicado en la planta baja de la Universidad, funciona como hospital-escuela y cuenta con salas comunes de internación, sala de terapia intermedia, unidad coronaria, servicio de neonatología y farmacia. 
"Experiencias de docencia, extensión e investigación para promover

la lactancia y la donación de leche materna"

Carla Estefanía Martin, Carina Lorena Fernández y Mara Cristina Romero

Vol. 21, Núm. 6, noviembre-diciembre 2020

Revista Digital Universitaria

Además de la prestación del servicio de salud, la ume cuenta con un camión sanitario cuya misión es ofrecer atención en forma integral a personas carentes de recursos, intentando asíllegar a zonas donde muchas veces es inaccesible el servicio de salud. El compromiso institucional con la formación de profesionales busca no solo la excelencia académica en los contenidos y metodología de enseñanza- aprendizaje, sino también en la conjunción de los objetivos académicos de las profesiones que intervienen en el proceso de salud-enfermedad-atención, con las necesidades de salud de la comunidad, entendiéndolos como un conjunto integrado e indisoluble. Es así que, cada salida del camión se organiza contemplando actividades en las que también participen los alumnos de las carreras del área de la salud (Farmacia, Licenciatura en Nutrición y Medicina); por ejemplo, los alumnos de los primeros años de Medicina, reciben a las personas y las derivan (dentro del camión) con el profesional correspondiente. Por su parte, los alumnos avanzados de Licenciatura en Nutrición, se encargan distribuir información sobre alimentación saludable y tomar las medidas antropométricas, para informar sobre índice de masa muscular y obesidad. Y de cualquiera de las dos carreras, revelan datos que puedan ser de interés sirviendo como de punto de partida para el diseño de estrategias de intervención en pos de la promoción de la salud.

\section{Desarrollo}

Las actividades que se describirán fueron realizadas durante los meses de marzo a noviembre del año 2018, integrando prácticas de extensión, docencia e investigación, en el marco de un Proyecto de Extensión Socio Comunitario (PESC) denominado "Lactancia materna y donación de leche". Como se indicó en el apartado anterior, las carreras del área de salud de nuestra universidad contemplan actividades que fomentan el contacto con los problemas de la sociedad, como las salidas con el camión sanitario. Es justamente en esas salidas donde pudo evidenciarse la baja prevalencia de lactancia materna, ya que, es frecuente observar que, durante el tiempo que esperan las madres con sus niños para recibir la atención, los infantes son alimentados con biberón, incluso aquellos que están en edad de recibir LME. Este comportamiento también es observado en las salas de espera de la UME, lo que hizo suponer que era necesario realizar intervenciones informativas mostrando los beneficios de la LME, y la utilidad de ésta para los recién nacidos que no pueden recibirla, para lo cual, la donación de leche materna se vuelve indispensable.

Con base en lo anterior, se conformó un grupo de trabajo, constituido por docentes investigadores de la carrera de Ingeniería en Alimentos de la universidad; docentes profesionales (médicos y nutricionistas) de las carreras de Medicina y la Licenciatura en Nutrición y alumnos de las carreras de Medicina, Licenciatura en Nutrición e Ingeniería en Alimentos. La metodología seguida fue la conveniente para indagar sobre conocimientos referidos a lactancia materna y BLm y realizar charlas informativas sobre estos mismos temas. En detalle, las actividades desarrolladas fueron las siguientes: 


\section{Identificación del problema}

Como se indicó anteriormente, la baja prevalencia de LME fue observada en las diferentes actividades realizadas por los alumnos.

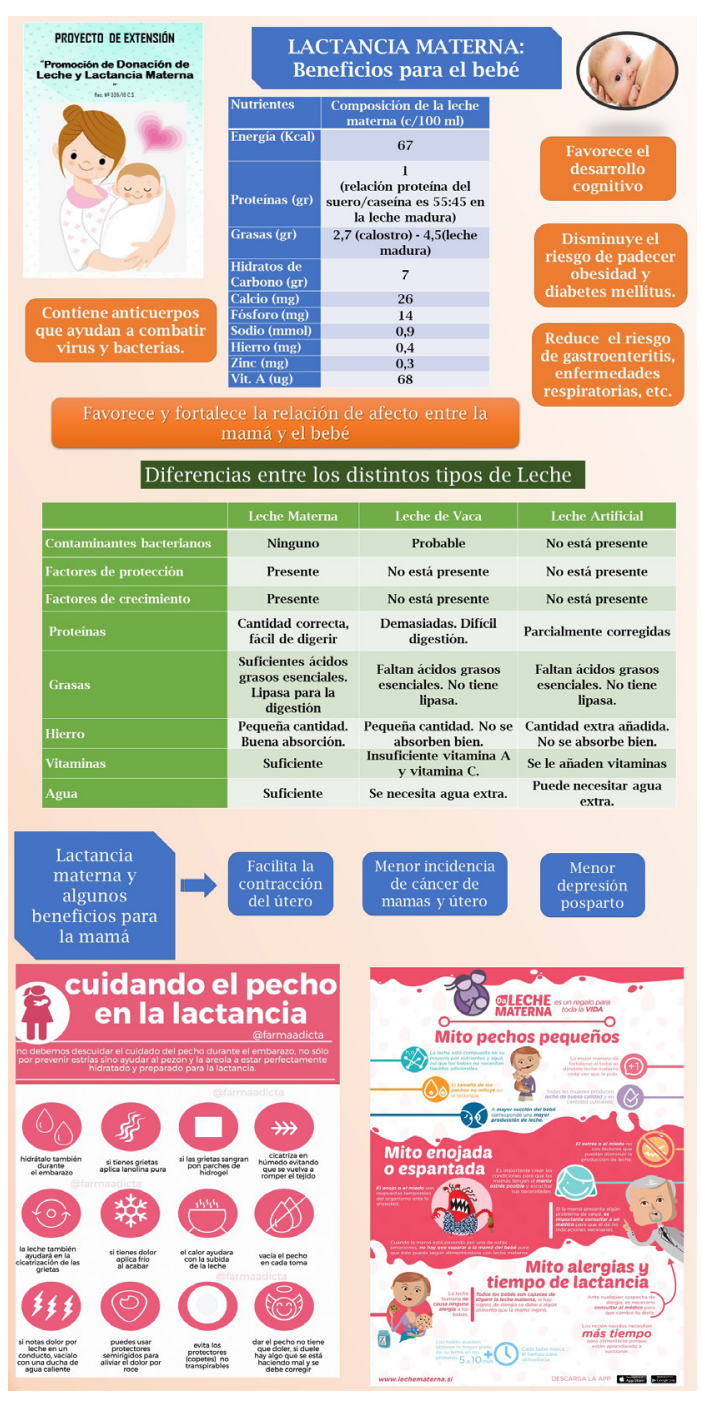

Asignación de tareas a cada integrante del grupo

Los docentes investigadores tuvieron a su cargo los aspectos metodológicos inherentes al diseño y validación del cuestionario destinado a indagar sobre los conocimientos y actitudes de la lactancia materna en madres, con la intención de identificar las causas de la baja prevalencia de leche materna. El cuestionario también incluyó preguntas que permitieron evaluar conocimientos y creencias respecto de la donación de leche materna y de la importancia de los bancos de leche humana. Los alumnos de las carreras involucradas tuvieron a su cargo la confección de material informativo (pósters, banners, folletos) y la recolección de datos. Los docentes de Medicina también se dedicaron a la recolección de datos (mediante el cuestionario mencionado anteriormente) aprovechando la asistencia a consulta.

Los docentes de Licenciatura en Nutrición supervisaron la confección del material educativo referido al problema de investigación, a partir del cual se elaboraron dos banners que también se imprimieron como folletos para su distribución en las salidas de campo. Uno de ellos presentaba información sobre los beneficios nutricionales e inmunológicos de la leche materna para el bebé, y también los beneficios para la madre, mostrando la influencia de la lactancia materna en el menor sangrado posparto y la rápida involución uterina posparto como se muestra en la figura 2 a) y b). Del mismo modo se elaboraron folletos con los mitos sobre la lactancia materna y donación de leche humana resaltando el interés de la misma, como se muestra en la figura 3. Los docentes de Licenciatura en Nutrición también tuvieron a su cargo la capacitación de los alumnos encargados de la recolección de datos y la realización de charlas informativas.

En estas charlas se brindó información complementaria a la de los folletos, se trabajó con pósteres y juegos sobre la posición correcta para amamantar, juegos de verdadero o falso para desvanecer mitos sobre lactancia materna, y se brindó información sobre cuidado de la mama y de la salud a través de una alimentación saludable. Para cerrar cada jornada, se realizó la promoción del Lactario y el BLm que funcionará en la UME, remarcando que los BLM son un servicio especializado, sin fines
Figura 2. Folletos empleados durante las charlas realizadas en las salidas a terreno, a) Informativo sobre lactancia materna, b) informativo sobre donación y extracción de leche. Fuente: elaboración propia. 


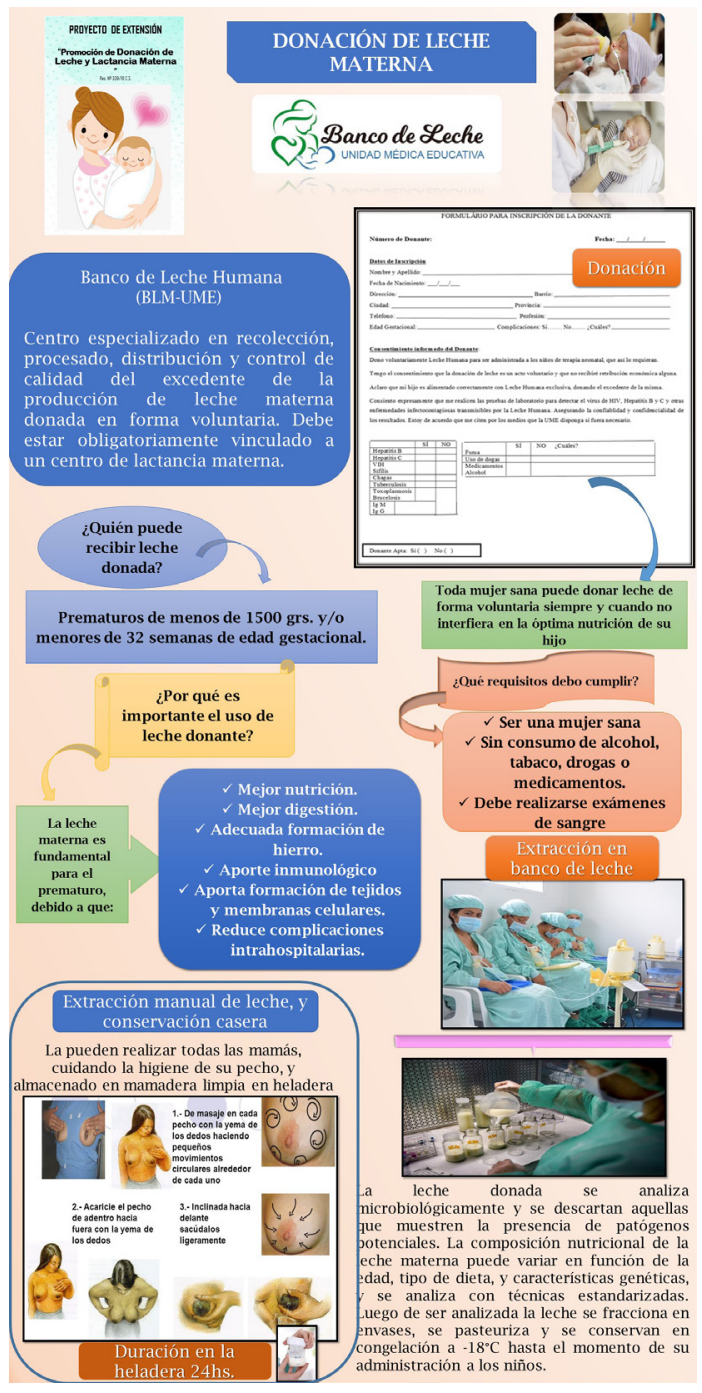

de lucro, responsable de la promoción, protección y apoyo a la lactancia materna y de la recolección, procesamiento y distribución de leche humana con calidad certificada. Al finalizar cada encuentro, se invitó a las asistentes a reflexionar sobre los conocimientos impartidos, para detectar errores en la intervención y establecer así acciones de mejora.

\section{Selección de puntos de recolección de datos}

La recolección de datos se realizó en tres ámbitos: las salidas del camión sanitario, las consultas médicas a los profesionales y las charlas informativas. En todos los casos la muestra fue por conveniencia, ya que se entrevistó a la totalidad de madres que estuvieron presentes en cada una de las instancias mencionadas durante todo el periodo de duración del proyecto. En este periodo se realizaron cuatro salidas del camión sanitario, veinte consultas médicas en consultorio y seis charlas informativas, las que se realizaron en seis Centros de Atención Primaria de Salud (CAPS) de nuestra ciudad.

\section{Resultados}

Respecto de los conocimientos sobre LME, sobre un total de 150 encuestas, el 92\% de las madres reconoció la importancia de la lactancia materna para el desarrollo integral del niño, mientras que el 59\% indicó que el término LME hace referencia a que lo único que puede ingerir el bebé es leche proveniente de la madre. Por otra parte, el $69 \%$ reconoció que la duración de la LME debe extenderse de forma exclusiva hasta los 6 meses, pero sólo el 23\% manifestó saber que se puede extender hasta los 2 años. Respecto del horario entre tomas, el 76\% de las entrevistadas indicó que ésta debe ser a libre demanda. Al ser cuestionadas sobre los beneficios brindados de la Lme para el bebé, el 52\% señaló que la LME sirve de protección contra diarrea y otras enfermedades, el 37\% indicó la prevención de la obesidad y otras enfermedades crónicas en edad adulta como uno de los beneficios, mientras que el 52\% indicó que el único beneficio de la LmE es que ayuda al niño a crecer saludablemente, sin identificar de manera específica los beneficios individuales. Al evaluar las respuestas a preguntas que indagaban sobre donación de leche humana y BLM, se detectó que el $81 \%$ de las madres conocían el hecho de poder donar leche materna, reconociendo el $43 \%$ que ésta es consumida por niños que nacen prematuros y que deben permanecer en el servicio de neonatología; resultó Ilamativo el alto porcentaje de madres (25\%) que manifestó no saber quiénes son los beneficiarios de esta donación. Aunque, el 65\% de las encuestadas 
Figura 3. Folleto informativo sobre lactancia materna. Fuente elaboración propia.

estuvo familiarizada con el término BLm o lactario, sólo el 27\% supo de la existencia de uno en nuestra provincia. Un porcentaje similar (23\%) indicó saber de la existencia del lactario que funciona en el hospital 4 de Junio como una extensión del BLm del Hospital Perrando, señalando que fueron usuarias del servicio de neonatología del hospital o bien conocen a algún familiar o vecino que lo fue. Por último, el 95\% de la población encuestada consideró importante la creación e implementación de un Banco de Leche Materna en nuestra ciudad.

\section{Lactancia Materna MITOS}

* Después de los 6 meses, la leche no está buena.

* Los bebés que se alimentan del pecho no engordan y siempre tienen hambre.

* Si das de mamar, no podrás ir a trabajar.

* Dar el pecho es doloroso y se te agrietan los pezones.

* Las madres con pechos pequeños no producen buena leche.

\section{PROYECTO DE EXTENSIÓN "Lactancia Materna y Donación de Leche"} Res. № 038 / 18 C.S.

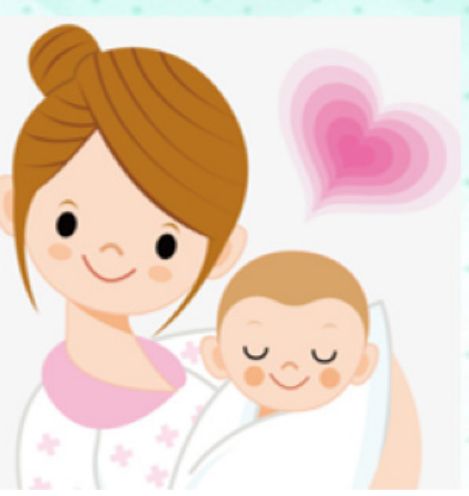

Respecto de las experiencias de los estudiantes que participaron en las actividades, la mayoría destacaron las siguientes como ventajas de realizar este tipo de actividades, por ejemplo:

- el conocimiento de las diferentes realidades sociales de la población local y de sus necesidades particulares,

- la interacción con distintos profesionales de la salud, docentes e investigadores,

- las oportunidades para mejorar y profundizar sus conocimientos en distintos temas,

- la mejora de sus habilidades de observación y capacidades de transmisión de conocimientos a distintos actores sociales, y por ultimo,

- la interacción y debate entre las prácticas y estrategias a utilizar, tanto con el equipo docente como con sus compañeros.

Sin embargo, y como era de esperarse, también surgieron algunos aspectos negativos durante las salidas a terreno, entre ellos, la desmotivación generada por las dificultades que se presentaron y la inconformidad al no poder 
implementar todas las propuestas o acciones a desarrollar y tiempo de duración de la práctica, al igual que lo relatado por Ambrústolo et al., (2018). Los resultados encontrados dieron origen a la propuesta de la puesta en marcha de un BLm en la umE y a un proyecto de investigación en el que se evaluarán alternativas de conservación de la leche materna recibida de las donantes, por lo que se espera que el BLm se convierta en un actor importante en materia de salud perinatal para las comunidades académico-científicas y sanitarias locales y nacionales.

\section{Conclusiones}

Los resultados obtenidos sugieren la necesidad de un mayor número de prácticas de intervención en pos de generar conciencia en la población sobre los temas tratados en este artículo. Además, se debe destacar que las actividades comunitarias realizadas, dieron origen a dos nuevos proyectos, uno de investigación y otro de extensión socio-comunitario, ambos iniciados a partir de marzo de 2019. Incrementar los conocimientos de la salud en la población es posible a través de la ejecución de jornadas o talleres informativos sobre lactancia materna, tanto para reforzar los conocimientos que ya la población posee como para corregir las carencias detectadas y prevenir el abandono de la misma. Esta intervención no debe estar destinada únicamente a las madres, ya que el acompañamiento del entorno familiar es de suma importancia durante el periodo de lactancia, siendo, de hecho, la falta de apoyo una de las causas frecuentes de abandono. Pudiéndose profundizar en conocimientos sobre donación de leche y funciones de un BLm, ya que la cuestión geográfica ubica a nuestra localidad en un punto estratégico para la puesta en marcha de un centro de estas características. Con la continuidad de este tipo de prácticas, la universidad conseguiría generar responsabilidad social universitaria en todos los integrantes de los proyectos de extensión, buscando transformar la comunidad con acciones planificadas que incluyan en forma conjunta los aspectos académicos, la investigación, la intervención y el conjunto de saberes, habilidades y destrezas inherentes a la disciplina de la unidad académica en la que se generen los proyectos. Esto resulta especialmente importante para los alumnos, ya que la formación de los profesionales no debe limitarse únicamente a los saberes disciplinares, sino que también debe apuntar a una formación global como ser humano que integra una sociedad y que por lo tanto requiere cultivar su compromiso civil. Por otro lado, las problemáticas a abordar con dichos proyectos deben buscarse de manera conjunta con la comunidad destinataria y en su propio terreno, por lo que la comunidad podrá visualizar de manera directa el impacto de la universidad dentro de la sociedad, reconociendo el rol de la misma en la promoción de la salud. 


\section{Referencias}

* Ambrústolo, M., Migueles, M., Berardi, M. y Zárate, C. (2018). Interacción entre la extensión y la investigación acción para el abordaje de una problemática en el sector productivo marplatense. +E: Revista de Extensión Universitaria, 8(9), 195 212. Dol: https://doi.org/10.14409/extension.v8i9.Jul-Dic.7856

* Aquino Rojas, E. E. (2018). Tácticas para la prevención de la desnutrición en niños menores de 6 meses a través de la concientización de la lactancia materna exclusiva en la población que asiste al Centro De Salud Universitario Norte De La Ciudad De Santa Cruz. Revista científica de salud UNITEPC, 4(1):21-9. https:// investigacion.unitepc.edu.bo/revista/index.php/revista-unitepc/article/view/30

* Delgado Panta, A. (2011). Organización para la implementación del Banco de Leche Humana en el área de neonatología del Hospital Rafael Rodríguez Zambrano de Manta marzo a agosto de 2011 [tesis para la obtención de Máster en Gerencia de Salud Para el Desarrollo Local, Manta, Manabí, Ecuador].

* Flores Antón, B., Alonso Díaz, C., y García Lara, N. R. (2015). Cómo se pone en marcha y cómo funciona un banco de leche. Experiencia del Hospital 12 de Octubre. Revista Española de Pediatría, 71(5), 301-303.

* Hernández Pérez, M. C., Díaz-Gómez, N. M., Romero Manzano, A. M., Díaz Gómez, J. M., Rodríguez Pérez, V. y Jiménez Sosa, A. (2018). Eficacia de una intervención para mejorar conocimientos y actitudes sobre lactancia materna en adolescentes. Revista Española de Salud Pública, 92, e201806033. http://ref.scielo.org/jdfv9g

* Herrera, M., Berganza, E., Giménez, S., Cardozo, D. y Jiménez, V. (2013). Puesta en marcha del primer Banco de Leche Humana en el Hospital Materno-Infantil San Pablo, Asunción, Paraguay. Pediatría (Asunción), 40(3), 353-260. https://www. revistaspp.org/index.php/pediatria/article/view/119

* Honorable Cámara de Diputados de la Nación Argentina. (2018). Programa de bancos de leche materna. Creación. https://www.hcdn.gob.ar/proyectos/proyecto. jsp?exp=6635-D-2018

* Injante Injante, M. A., Alvarez Diaz, G. A., Gavilano Bernaola, L. M. y Macera Barriga, C. (2017). Conocimientos, actitudes y prácticas de lactancia materna de madres que acuden al control pre-natal en un hospital de Ica-Perú. Revista Médica Panacea, 6(2): 53-59. https://doi.org/10.35563/rmp.v6i2.51

* Kramer, M. S. y Kakuma, R. (2001). The Optimal Duration of Exclusive Breastfeeding: A Systematic Review. World Health Organization. https://www.who.int/nutrition/ publications/optimal_duration_of_exc_bfeeding_review_eng.pdf

* López, M. L. (2016). Extensión universitaria y salud pública en Argentina como caso testigo en la región. Revista de Investigación Educativa, 34(1), 119-132. http:// www.redalyc.org/articulo.oa?id=283343416007

* Marín, G. (2017). Extensión universitaria: un enfoque desde el campo de la salud. Masquedós, 2(2): 43-52. http://sedici.unlp.edu.ar/bitstream/ handle/10915/103446/.pdf-PDFA.pdf?sequence=1\&isAllowed=y 
* Ministerio de Salud y Desarrollo Social. Presidencia de la Nación. (2015). Reglamentan la ley que promueve la lactancia materna. http://www.msal.gob. ar/prensa/index.php/noticias-de-la-semana/2494-reglamentan-la-ley-quepromueve-la-lactancia-materna

* Ministerio de Salud de la Nación. (2015). Dirección Nacional de Maternidad, Infancia y Adolescencia. Situación de la lactancia materna en Argentina.

* Ministerio De Salud de la República Argentina. (2015). Resolución 270/2015. http:// servicios.infoleg.gob.ar/infolegInternet/anexos/245000-249999/246449/norma. htm

* Ministerio de Salud de la Nación. (2018). Dirección Nacional de Maternidad, Infancia y Adolescencia. Situación de la lactancia materna en Argentina.

* oms/Unicef/Usaid. (2008). Indicadores para evaluar las prácticas de alimentación del lactante y del niño pequeño. Organización Mundial de la Salud.

- Sager, G. (2018). Centros de Lactancia Materna y Bancos de Leche Humana. https:// www.sap.org.ar/docs/congresos_2018/Lactancia/Sager_centrolactanciamaterna.pdf

\section{Cómo CITAR ESTE ARTículo}

* Martin, Carla Estefanía, Fernández, Carina Lorena y Romero, Mara Cristina. (2020, noviembre-diciembre). Experiencias de docencia, extensión e investigación para promover la lactancia y la donación de leche materna. Revista Digital Universitaria (RDU), 21(6). Dol: http://doi.org/10.22201/cuaieed.16076079e.2020.21.6.6 